\title{
NUMERICAL SOLUTION TO BOUNDARY LAYER PROBLEM OF NON-NEWTONIAN FLUID FLOW OVER A MOVING SURFACE
}

\author{
Gabriella Bognár and Zoltán Csáti \\ Department of Machine and Product Design, University of Miskolc \\ 3515 Miskolc, Miskolc-Egyetemváros, Hungary \\ v.bognar.gabriella@uni-miskolc.hu, machcsz@uni-miskolc.hu
}

[Received: June 01, 2015, Accepted: October 26, 2015.]

Dedicated to Professor Barna Szabó on the occasion of his eightieth birthday

\begin{abstract}
The paper generalizes the classical Sakiadis problem for non-Newtonian power law fluids. Applying the similarity method the governing partial differential equations are transformed to one ordinary differential equation. The resulting boundary value problem is solved by Chebyshev spectral collocation on a truncated domain. The effect of the power-law exponent on the numerical solutions is investigated.
\end{abstract}

Mathematical Subject Classification: 34B40, 65N35

Keywords: non-Newtonian fluid, boundary layer, similarity method, spectral method

\section{INTRODUCTION}

The study of a flow generated by a moving surface in an otherwise quiescent fluid plays a significant role in many material processing applications such as hot rolling, metal forming and continuous casting (see e.g., Altan et al. [1], Fisher [2, Tadmor and Kline [3]). Boundary layer flow induced by the uniform motion of a continuous plate in a Newtonian fluid has been analytically studied by Sakiadis [4] and experimentally by Tsou et al. 5]. A polymer sheet extruded continuously from a die travelling between a feed roll and a wind-up roll was investigated by Sakiadis 6], 44. For the laminar velocity field of a Newtonian fluid Tsou et al. 5 showed in their analytical and experimental study that the obtained analytical results are in excellent agreement with the measured data, therefore it proves that the mathematical model for boundary layer on a continuous moving surface describes a physically realizable flow.

Recently, a number of researchers are motivated to investigate the problem of boundary layer flow due to its application in engineering processes. Flows along a continuously moving surface are encountered in several processes, for example the thermal and moisture treatment of materials, particularly in processes involving continuous pulling of a sheet through a reaction zone, in metallurgy, the paper industry, 
and in the manufacture of polymeric sheets. Fluids for which the relationship between the shear stress and the rate of strain is non-linear at given temperature and pressure are said to be non-Newtonian. Most fluids such as molten plastics, artificial fibres, petroleum, blood and polymer solutions are considered as non-Newtonian fluids. Schowalter 7] introduced the concept of the boundary layer in the theory of non-Newtonian power-law fluids. Acrivos, Shah and Petersen [8] have investigated the steady laminar flow of non-Newtonian fluids over a plate.

Our aim is to examine the solutions to the boundary layer problem of a power-law non-Newtonian fluid along an impermeable flat surface moving with a constant velocity in an otherwise quiescent fluid environment. In the absence of an exact solution in closed form, numerical solutions for the velocity distribution in the boundary layer for different power-law exponents will be presented, and the dependence of the skin friction parameter and the boundary layer thickness on the power-law exponent $n$ are examined.

In this paper, we apply a spectral method for the solution to the boundary value problem (BVP). Spectral methods were first applied in the 1970s but their mathematical foundation were endowed by Gottlieb and Orszag in 1977. By the 1990s, spectral methods had become attractive tools for scientists dealing with fluid mechanics and meteorological modeling 9]. In contrast to the finite difference or the finite element methods which are local in character, spectral methods are global methods. With the advent of the spectral element method, complicated domains can be handled. In spite of being mainly used in fluid mechanics, nowadays, they are more and more frequently utilized in biomechanics, astrophysics and the study of electromagnetic waves.

The text is organized into four sections. In Section 2 the governing equations describing the phenomenon are introduced. In Section 3 a BVP of nonlinear ordinary differential equations is derived by applying the similarity method. Section 4 is devoted to the numerical solution of the BVP using a spectral collocation method. Finally, we summarize the consequences of our investigations in Section 5

\section{Governing Equations}

Consider a steady, two-dimensional, laminar boundary layer flow of viscous, incompressible power-law fluid past a continuously moving plate passing through with constant velocity $U_{w}$ in an otherwise quiescent fluid. The $x$-axis extends parallel to the plate and the $y$-axis is perpendicular to the $x$-axis. The flat surface is placed at $y=0$. The boundary layer equations for a flow over a flat plate neglecting pressure gradient and body forces can be described by the following continuity and momentum equations [5]:

$$
\begin{gathered}
\frac{\partial u}{\partial x}+\frac{\partial v}{\partial y}=0 \\
u \frac{\partial u}{\partial x}+v \frac{\partial u}{\partial y}=\frac{1}{\rho} \frac{\partial \tau_{y x}}{\partial y}
\end{gathered}
$$


where $u$ and $v$ denote the horizontal and vertical fluid velocity component, respectively, and $\tau_{y x}$ is the shear stress. Hereafter, we apply the Ostwald-de Waele powerlaw constitutive equation for the non-Newtonian model [10]:

$$
\tau_{y x}=K\left|\frac{\partial u}{\partial y}\right|^{n-1} \frac{\partial u}{\partial y},
$$

where $K$ is the consistency and $n$ is the power-law exponent. If $n<1$, the fluid is pseudoplastic, if $n>1$ it is dilatant while the fluid is Newtonian for $n=1$. Substituting Eq. 2.3 into 2.2), the fundamental equation is obtained for the velocity field. Equation (2.1) can identically be satisfied when the stream function $\psi$ is introduced as $u=\partial \psi / \partial y, v=-\partial \psi / \partial x$. Then from 2.3 we get a third order nonlinear partial differential equation:

$$
\psi_{y} \psi_{y x}-\psi_{x} \psi_{y y}=\gamma\left(\left|\psi_{y y}\right|^{n-1} \psi_{y y}\right)_{y}
$$

where $\gamma=K / \rho$ and the subscripts denote the partial differentiation with respect to the appropriate variable. The wall is impermeable and no-slip boundary condition is supposed. Furthermore, the ambient fluid velocity is zero and we suppose that the plate is moving at a constant velocity; therefore the boundary conditions can be formulated as follows:

$$
u(x, 0)=U_{w}, \quad v(x, 0)=0, \quad \lim _{y \rightarrow \infty} u(x, y)=0 .
$$

\section{Similarity SOLUtion}

The similarity method is applied for the transformation of 2.4 to an ordinary differential equation. Let us introduce similarity variables $\eta$ and $f(\eta)$ as

$$
\eta=a \frac{y}{x^{\beta}}, \quad \psi=b x^{-\alpha} f(\eta)
$$

where $a, b, \alpha, \beta$ are constants, which will be determined from the invariance condition for the differential equation and the three boundary conditions. Substituting (3.1) into Eq. 2.4 one obtains

$$
a^{2} b^{2} x^{-2 \alpha-2 \beta-1}\left[a f f^{\prime \prime}-(\alpha+\beta) f^{\prime 2}\right]=\gamma\left(a^{2} b\right)^{n} x^{(-\alpha-2 \beta) n} \frac{a}{x^{\beta}}\left[\left|f^{\prime \prime}\right|^{n-1} f^{\prime \prime}\right]^{\prime},
$$

where the primes denote differentiation with respect to $\eta$. One can observe that the differential equation remains invariant if

$$
(2 n-1) \beta+(n-2) \alpha=1
$$

and

hold.

$$
a^{2} b^{2}=\gamma\left(a^{2} b\right)^{n} a
$$

Rewriting the boundary conditions by applying the stream function, one may establish other connections among the parameters. According to (3.1), in the third condition of 2.5 if $y \rightarrow \infty$, i.e. $\eta \rightarrow \infty$, for fixed $x$ we get

$$
\lim _{y \rightarrow \infty} \psi_{y}(x, y)=\lim _{\eta \rightarrow \infty} f^{\prime}(\eta)=a b x^{-\alpha-\beta} f^{\prime}(\eta)=0 .
$$


Let us choose $f^{\prime}(\infty)=\lim _{\eta \rightarrow \infty} f^{\prime}(\eta)=0$, then

$$
\alpha+\beta=0 .
$$

At $\eta=0$ setting

$$
f^{\prime}(0)=1
$$

implies

$$
a b=U_{w}
$$

from the first condition in 2.5. On the other hand, from Eq. 3.2 and from Eq. (3.4) it follows that

$$
\beta=-\alpha=\frac{1}{n+1} .
$$

The second condition in 2.5 is expressed as

$$
-\psi_{x}(x, 0)=\frac{b}{n+1} x^{-\frac{n}{n+1}}\left[\eta f^{\prime}(\eta)-f(\eta)\right]=0,
$$

which is satisfied for $\eta=0$ if

$$
f(0)=0
$$

Consequently, the parameter values are the following:

$$
\begin{aligned}
\alpha=-\frac{1}{n+1}, & \beta=\frac{1}{n+1}, \\
a=\gamma^{-\frac{1}{n+1}} U_{w} \frac{2-n}{n+1}, & b=\gamma^{\frac{1}{n+1}} U_{w}^{\frac{2 n-1}{n+1}} .
\end{aligned}
$$

The connection among the dimensionless similarity variables and the original velocity is

$$
\begin{gathered}
u(x, y)=U_{w} f^{\prime}(\eta), \\
v(x, y)=\frac{U_{w}}{n+1} R e_{x}^{-\frac{1}{n+1}}\left(\eta f^{\prime}(\eta)-f(\eta)\right), \\
\eta=R e_{x}^{\frac{1}{n+1}} \frac{y}{x}, \quad R e_{x}=\frac{U_{w}^{2-n} x^{n}}{\gamma},
\end{gathered}
$$

where $R e_{x}$ is the local Reynolds number. Instead of the BVP of partial differential equations $(2.1),(2.2),(2.3)$ and $(2.5)$ we obtained by the similarity analysis the BVP of a nonlinear ODE (3.9) and 3.10)

$$
\begin{gathered}
\left(\left|f^{\prime \prime}\right|^{n-1} f^{\prime \prime}\right)^{\prime}+\frac{1}{n+1} f f^{\prime \prime}=0 \\
f(0)=0, \quad f^{\prime}(0)=1, \quad \lim _{\eta \rightarrow \infty} f^{\prime}(\eta)=0 .
\end{gathered}
$$

Since the velocity field can be calculated from $f(\eta)$ using (3.6)-(3.7), our goal is to determine the solution to $3.9-3.10$. We also want to examine the boundary layer thickness and $f^{\prime \prime}(0)$. The latter one is necessary for computation of the drag coefficient

$$
C_{D}=(n+1)^{\frac{1}{n+1}} R e^{-\frac{n}{n+1}}\left(-f^{\prime \prime}(0)\right)^{n}
$$

and the wall shear stress

$$
\tau_{w}(x)=\left[\frac{\varrho^{n} K U_{w}^{3 n}}{x^{n}}\right]\left(-f^{\prime \prime}(0)\right)^{n} .
$$




\section{Numerical Solution}

We use a spectral method for the determination of the solution to $3.9-(3.10)$. Spectral methods are able to provide very accurate results when the solution is smooth enough. More precisely, if the solution is differentiable to all orders, an exponential (or infinite order or spectral) convergence is achieved. However, if the solution is $m$-times continuously differentiable, the rate of convergence is algebraic: $\mathcal{O}\left(k^{-m}\right)$, where $k$ is the $k$-th expansion mode [11. Superior convergence can be achieved for entire functions. For periodic problems, Fourier spectral methods are straightforward; however, in our case the domain is non-periodic, therefore we are going to use the Chebyshev method. All three versions of spectral methods (collocation, Galerkin and tau) belong to the method of weighted residuals and the main classification is carried out according to the type of trial functions used. Trial functions in the Galerkin method are the same as the weight functions and satisfy some of the boundary conditions. In spectral collocation, trial functions are Dirac-delta functions located at the collocation points while the tau method, similarly to the Galerkin method, operates in the weak form but the trial functions generally do not satisfy the boundary conditions [9]. In our calculations the collocation method is used. During collocation we determine the function values of the interpolating polynomial at the collocation points (nodal approximation) as opposed to the other two methods, which give results for the coefficients of the truncated approximating series (modal approximation). For other aspects of the method, we refer to 9 .

The $n$-th order Chebyshev polynomial of the first kind, $T_{n}(\bar{x})$ is defined on $[-1,1]$ and can be expressed by the recursion

$$
T_{0}(\bar{x})=1, \quad T_{1}(\bar{x})=\bar{x}, \quad T_{n}(\bar{x})=2 \bar{x} T_{n-1}(\bar{x})-T_{n-2}(\bar{x}), n>1 .
$$

The modal approximation of a function $w(\bar{x})$ is

$$
I_{N} w(\bar{x})=\sum_{j=0}^{N} a_{j} T_{j}(\bar{x}),
$$

where $a_{j}$ are the Chebyshev coefficients. The nodal approximation of $w(\bar{x})$ can be evaluated in the Lagrange base as

$$
p_{N} w(\bar{x})=\sum_{j=0}^{N} w_{j} \ell_{j}(\bar{x}),
$$

where $\ell_{j}$ are the Lagrange basis polynomials. The spectral differentiation for Chebyshev polynomials can be carried out either by a matrix-vector product or by using the Fast Fourier Transform (FFT). We implement the matrix-vector multiplication method because of the relatively low number of collocation points. The first derivative of $w$ is approximated as

$$
w^{\prime}\left(\bar{x}_{i}\right) \approx \sum_{j=0}^{N} D_{i j} w_{j}, \quad i=0, \ldots, N,
$$


where $\mathbf{D}$ is the first differentiation matrix. Similarly, the $p$-th order derivative is calculated as

$$
\frac{\mathrm{d}^{p} w\left(\bar{x}_{i}\right)}{\mathrm{d} \bar{x}^{p}} \approx \sum_{j=0}^{N} D_{i j}^{(p)} w_{j}, \quad i=0, \ldots, N,
$$

with $\mathbf{D}^{(p)}$ standing for the $p$-th differentiation matrix. For $\mathbf{D}$ and $\mathbf{D}^{(2)}$ exact formulas exist.

One of the methods for solving a BVP on an infinite or semi-infinite interval is the so-called domain truncation [11. Performing the truncation and the linear mapping we have

$$
\eta \in[0, \infty) \longrightarrow \xi \in[0, L] \stackrel{\zeta=\frac{\xi}{L}}{\longrightarrow} \zeta \in[0,1] \stackrel{\bar{x}=2 \zeta-1}{\longrightarrow} \bar{x} \in[-1,1] .
$$

Introducing $\bar{f}(\bar{x})=f(\eta(\bar{x}))$, BVP $3.9-3.10$ reads

$$
\begin{gathered}
\frac{8}{L^{3}} \bar{f}^{\prime \prime \prime}-\frac{1}{n(n+1)}\left(\frac{4}{L^{2}}\right)^{2-n} \bar{f}\left|\bar{f}^{\prime \prime}\right|^{2-n}, \\
\bar{f}(-1)=0, \quad \bar{f}^{\prime}(-1)=L / 2, \quad \bar{f}^{\prime}(1)=0 .
\end{gathered}
$$

After the discretization of $\bar{f}, N+2$ number of algebraic equations are at our disposal. The differential equation (4.5) is approximated at the $N-1$ inner nodes and the three boundary conditions (4.6). However, the number of unknowns are only $N+1$, therefore the resulting system is overdetermined. One possible solution is to take an interpolant that already satisfies some of the boundary conditions [12]. Let us seek function $g$ such that

$$
\bar{f}(\bar{x})=P(\bar{x}) g(\bar{x}), \quad P(\bar{x})=a \bar{x}^{2}+b \bar{x}+c .
$$

In case of $P(-1)=0, P^{\prime}(-1)=L / 2$ and $P^{\prime}(1)=0$ are satisfied, $a, b, c$ are obtained as

$$
a=-L / 8, \quad b=L / 4, \quad c=3 L / 8 .
$$

Now the differential equation is reformulated by

$$
\begin{gathered}
\frac{8}{L^{3}}\left[6 a g^{\prime}+(6 a \bar{x}+3 b) g^{\prime \prime}+\left(a \bar{x}^{2}+b \bar{x}+c\right) g^{\prime \prime \prime}\right]-\frac{1}{n(n+1)}\left(\frac{4}{L^{2}}\right)^{2-n}\left(a \bar{x}^{2}+b \bar{x}+c\right) g \\
\left|2 a g+(4 a \bar{x}+2 b) g^{\prime}+\left(a \bar{x}^{2}+b \bar{x}+c\right) g^{\prime \prime}\right|^{2-n}=0
\end{gathered}
$$

under boundary conditions

$$
g(-1)=1, \quad g^{\prime}(1)=0 .
$$

The BVP (4.7)- 4.8) can be solved with the Chebyshev spectral technique.

After the discretization of $g(\bar{x})$ and its derivatives according to $(4.2)$ and $(4.3)$, the resulting system of nonlinear equations is solved by the Levenberg-Marquardt algorithm in Matlab. Table 1 contains the essential values of $f^{\prime \prime}(0)$ for various number of collocation points and for different interval length $L$ and power-law exponent $n$.

The numerical results for different power-law exponents are depicted in Fig. 1 . where $f^{\prime}(\eta)$ is shown which is proportional to $u(x, y)$ (see Eq. (3.6)). The figure shows that with larger values of $n$ the boundary layer thickness decreases and shorter 
Table 1. Values of $f^{\prime \prime}(0)$ for different power-law exponents

\begin{tabular}{ccccc}
\hline & $n=0.5$ & $n=0.7$ & $n=0.8$ & $n=1$ \\
& $(L=30, N=23)$ & $(L=25, N=25)$ & $(L=20, N=20)$ & $(L=20, N=30)$ \\
\hline$f^{\prime \prime}(0)$ & -0.468917 & -0.443623 & -0.441151 & -0.443748 \\
\hline & $n=1.1$ & $n=1.3$ & $n=1.5$ & \\
\hline$f^{\prime \prime}(0)$ & -0.447283 & -0.45624 & -0.466919 & \\
\hline
\end{tabular}

interval is enough for the truncation. Because $f^{\prime \prime}(0)<0$, both the drag coefficient in

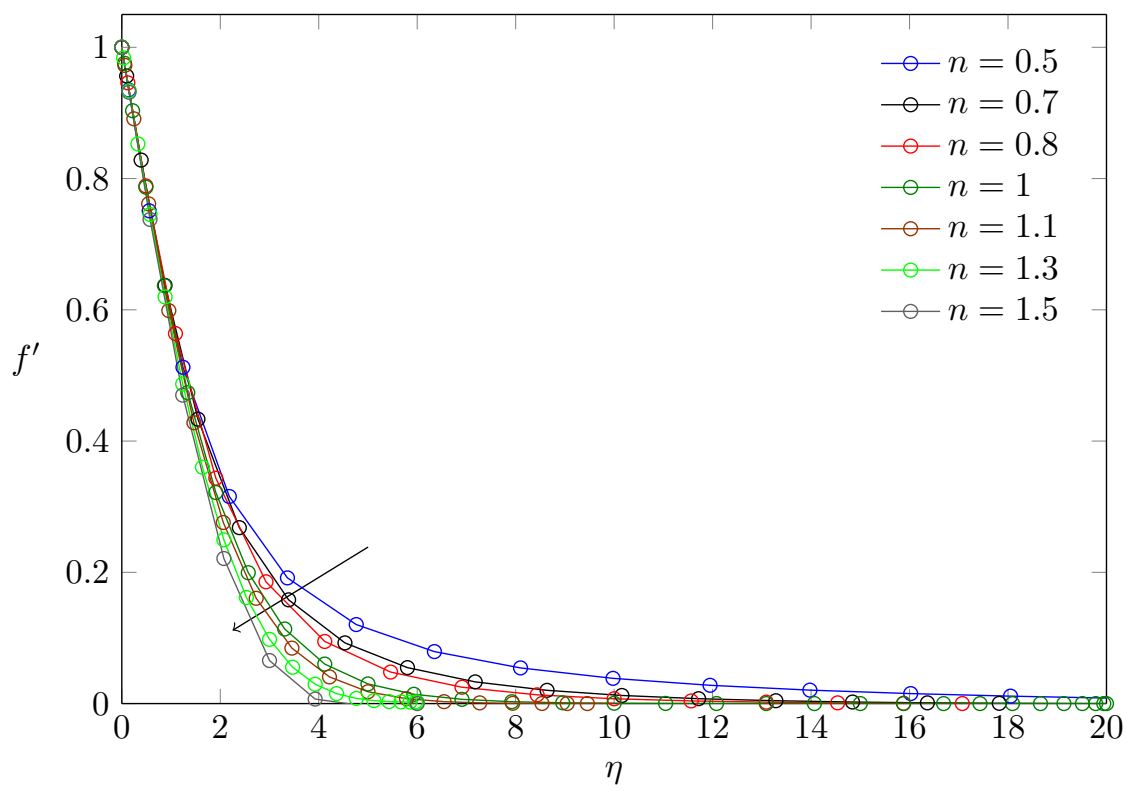

Figure 1. Velocity profiles for different values of $n$

Eq. 3.11 and the wall shear stress in Eq. 3.12 are influenced by $\left(-f^{\prime \prime}(0)\right)^{n}$ and this is described in Fig. 2 .

Table 2 collects the calculated function values for some $n$.

Error estimation is based on the monotonic decrease of the absolute value of the Chebyshev coefficients $a_{j}$. Namely, if the coefficients before $a_{N}$ tend to $a_{N}$ smoothly in absolute value, then the solution is supposed to be correct 11. The last retained coefficient $a_{N}$ in (4.1) is proportional to the truncation error. We also performed the calculations for different numbers of collocation points. For the calculated values of 


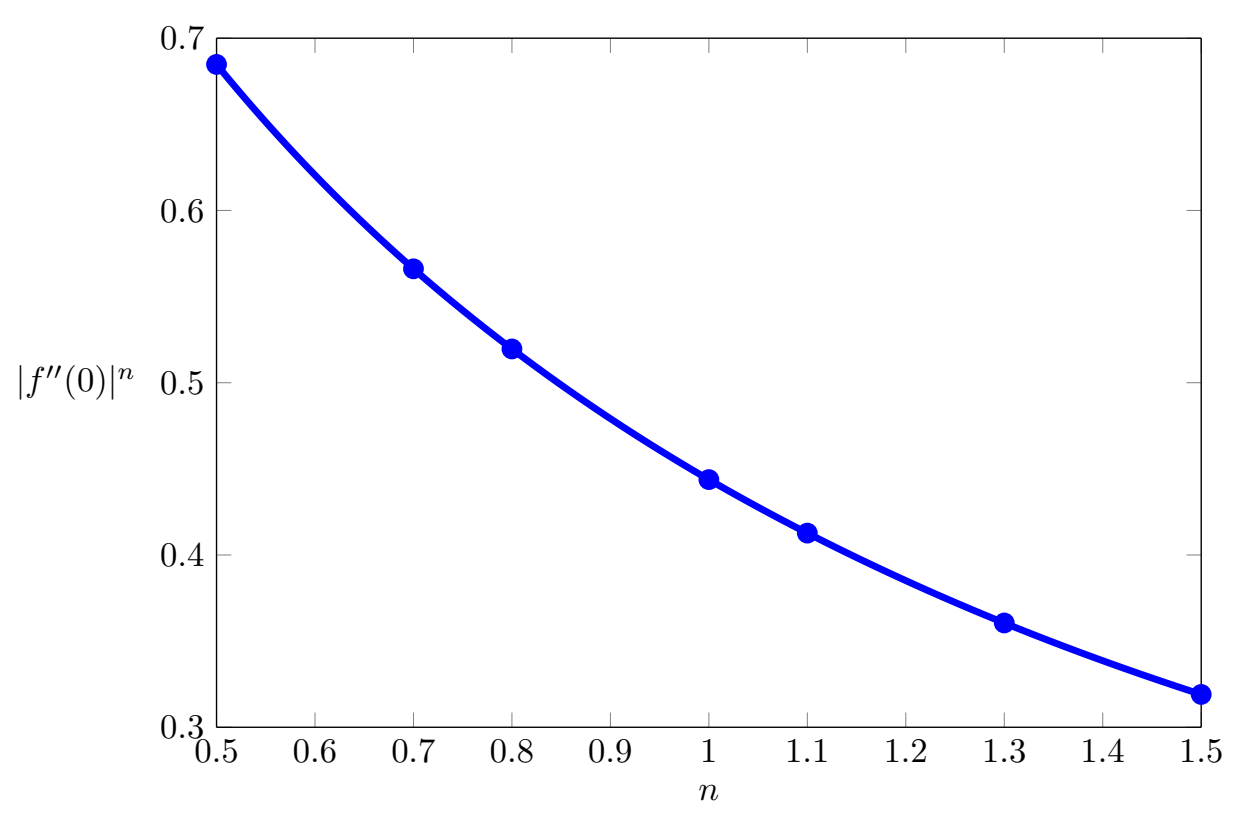

Figure 2. The values of $\left|f^{\prime \prime}(0)\right|^{n}$ for $n \in[0.5,1.5]$

Table 2. The values of $\left|f^{\prime \prime}(0)\right|^{n}$ for some values of $n$

\begin{tabular}{lcccc}
\hline$n$ & 0.5 & 0.7 & 0.8 & 1 \\
\hline$\left|f^{\prime \prime}(0)\right|^{n}$ & 0.684775 & 0.566122 & 0.519601 & 0.443763 \\
\hline$n$ & 1.1 & 1 & 1.5 & \\
\hline$\left|f^{\prime \prime}(0)\right|^{n}$ & 0.412706 & 0.360538 & 0.319053 & \\
\hline
\end{tabular}

$n$, using the values of $L$ and $N$ given in Table 1 , the worst estimated error is obtained for $n=0.5$ as $1.55 \mathrm{e}-6$ and the best one is $3.70 \mathrm{e}-11$ for $n=1$.

\section{Conclusions}

The paper deals with the solutions to the generalized Sakiadis problem for nonNewtonian power-law fluids. The boundary layer assumption is taken into account, then a similarity transformation is used in order to solve an ordinary differential equation instead of the system of partial differential equations. Chebyshev spectral collocation is applied after domain truncation and the appropriate treatment of the boundary conditions. From the solutions achieved, we found that the boundary layer thickness decreases as the power-law exponent increases, while the drag coefficient and the wall shear stress increase with larger $n$ exponents. 


\section{REFERENCES}

1. Altan, T., Оh, S., and Gegel, H.: Metal Forming Fundamentals and Applications. Asm. Intl., 1979.

2. Fisher, E. G.: Extrusion of Plastics. Wiley, New York, 1976.

3. Tadmor, Z. and Klein, I.: Engineering Principles of Plasticating Extrusion. Polymer Science and Engineering Series, Van Nostrand Reinhold, New York, 1970.

4. SAKIADIS, B. C.: Boundary layer behavior on continuous solid surfaces. II: The boundary layer on a continuous flat surface. AIChE, 7(2), (1961), 221-225.

5. Tsou, F., Sparrow, E., and Goldstein, R.: Flow and heat transfer in the boundary layer on a continuous moving surface. Int. J. Heat Mass Transfer, 10(2), (1967), 219-235.

6. SAKiadis, B. C.: Boundary-layer behavior on continuous solid surfaces: I. Boundarylayer equations for two-dimensional and axisymmetric flow. AIChE, 7(1), (1961), 26-28.

7. Schowalter, W.: Mechanics of Non-Newtonian fluids. Pergamon Press, 1st edn., 1978.

8. Acrivos, A., Shah, M., and Petersen, E.: Momentum and heat transfer in laminar boundary flow of non-Newtonian fluids past external surfaces. AIChE Journal, 6(2), (1960), 312-317.

9. Canuto, C., Hussaini, M. Y., Quarteroni, A., and Zang, T. A.: Spectral Methods: Fundamentals in Single Domains. Springer, 1st edn., 2006.

10. Pop, I. and Gorla, R.: Mixed convection similarity solutions of a non-newtonian fluid on a horizontal surface. Wärme-und Stoffübertragung, 26, (1990), 57-63.

11. Boyd, J. P.: Chebyshev and Fourier Spectral Methods. Dover Publications, 2000.

12. Fornberg, B.: A pseudospectral fictitious point method for high order initial-boundary value problems. SIAM J. Sci. Comput., 28(5), (2006), 1716-1729. 